\title{
Analysis of Nonlinear Damping Characteristic of Regenerative Damper
}

\author{
Songshan Liu ${ }^{\mathrm{a}}$, Qingnian Wang ${ }^{\mathrm{b}}$, Weihua Wang ${ }^{*} \mathrm{c}$ \\ ${ }^{1}$ State Key Laboratory of Automotive Simulation and Control \\ Jilin University, Changchun, Jilin, China \\ ajdvdlss08@126.com, bwqn@jlu.edu.cn, cwwh_jlu@126.com \\ ${ }^{*}$ Corresponding author
}

Keywords: regenerative damper, damping characteristic, vibration performance

\begin{abstract}
This paper presents an innovative damping characteristic of regenerative based on passive damper and permanent magnetic synchronous motor torque and speed characteristic. Different damping characteristic models were incorporated into single degree freedom of vehicle model and investigated. Simulation results show that the proposed regenerative damping characteristic is superior to others under harmonic excitation.
\end{abstract}

\section{Introduction}

The researched regenerative damper is based on a rotary permanent magnetic synchronous motor that enables continuous damping profile variation and control, allows implementation of passive, semi-active or fully active control scheme and regenerates the suspension vibration energy. The torque and speed of regenerative motor are converted to damping force and linear velocity by ball and screw nut [1].

Traditional vehicle suspension analysis theory was based on linear damper model assumed damper force versus speed curve is linear. However, the torque of regenerative motor is a constant before the rated speed called as constant torque region and after that, is inversely proportional to the speed until the max speed called as constant power region [2]. Hence it will encounter a serious problem when using the motor as regenerative damper. The damper force of regenerative motor is proportionally increased as linear damper until it reaches the max force of motor, then it will keep a constant damper force before rated speed of motor, after that the regenerative damping force will reduce as the velocity increases. Such regenerative damping character is similar to pressure-limited hydraulic damper as illustrated in literature [3], but more complicated. Therefore nonlinear damping characteristic of regenerative damper will be analyzed to evaluate the influence on vehicle performance.

Single degree of freedom vehicle mode is established and analyzed its amplitude-frequency characteristics. Different damper control schemes including passive linear model, asymmetrical model, semi-active on-off control model, and regenerative damper model will be incorporated to single degree of freedom (SDOF) vehicle mode and comparison will be made under harmonic vibration.

\section{SDOF Vehicle Model}

Figure 1(a) and 1(b) illustrate the model representation of a based excited single degree of freedom vehicle model employing passive and variable damping elements.

The equation of motion of SDOF vehicle model employing a linear spring and linear damper can be expressed as:

$$
\mathrm{m} \ddot{z}+c(\dot{z}-\dot{q})+k(z-q)=0
$$

Where, $m$ is the mass of sprung mass; $k$ is the spring stiffness; $c$ is the linear damping coefficient; $z$ is the displacement of sprung mass; $q$ is the harmonic base-excitation displacement. 


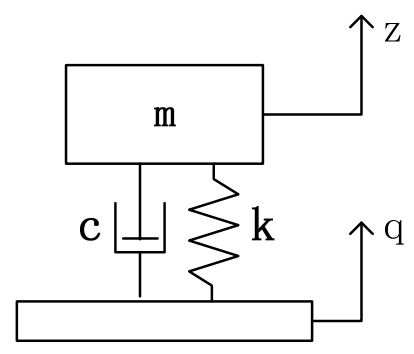

(a)

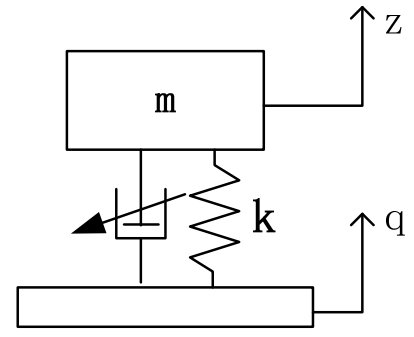

(b)

Fig. 1 SDOF vehicle model with (a) passive damper, and (b) variable damper

\section{Damper Control Schemes}

Damper force is directly related to the damper structures or control schemes.

For the passive damper, the damper force is proportional to the relative velocity, and can be expressed as follows:

$$
f_{d p}=c(\dot{z}-\dot{q})
$$

For asymmetrical passive damper, the damper force is related to the sign of relative velocity. When the damper is in compression stroke, the low damper force is exerted. In contrast, in extension stroke, high damper force is exerted.

$$
f_{d a}= \begin{cases}c(\dot{z}-\dot{q}) & \text { if }(\dot{z}-\dot{q})>0 \\ \lambda c(\dot{z}-\dot{q}) & \text { if }(\dot{z}-\dot{q})<0\end{cases}
$$

Where, $\lambda$ is the reduction coefficient when compression stroke.

For semi-active control scheme proposed by Karnopp et al. [4], the damper force is based upon the sign of the product of absolute velocity and relative velocity:

$$
f_{d s}= \begin{cases}c(\dot{z}-\dot{q}) & \text { if }: \dot{z}(\dot{z}-\dot{q})>0 \\ \lambda c(\dot{z}-\dot{q}) & \text { if }: \dot{z}(\dot{z}-\dot{q})<0\end{cases}
$$

For the regenerative damper mentioned in this paper, the damping characteristic is determined by the torque and speeds $(T-n)$ characteristic of regenerative motor and passive damper characteristic.

$$
f_{d r}= \begin{cases}c(\dot{z}-\dot{q}) & \text { if }:|\dot{z}-\dot{q}|<v_{1} \\ \operatorname{sgn}(\dot{z}-\dot{q})^{*} c v_{1} & \text { if }: v_{1} \leq|\dot{z}-\dot{q}| \leq v_{2} \\ c v_{1} v_{2} /(\dot{z}-\dot{q}) & \text { if }: v_{2}<|\dot{z}-\dot{q}|<v_{\max }\end{cases}
$$

As illustrated in Fig. 2, when the velocity of regenerative damper is under the $v_{1}$, the damping characteristic is the same as passive damper, otherwise, is the same as regenerative motor $F-v$ characteristic converted from the motor $T-n$ characteristic. The symbols $v_{2}, c v_{1}$ and $c v_{1} v_{2}$ are respectively corresponding to the motor rated velocity, max force and power, and the function of $\operatorname{sgn}(\dot{z}-\dot{q})$ is calculate the sign of relative velocity.

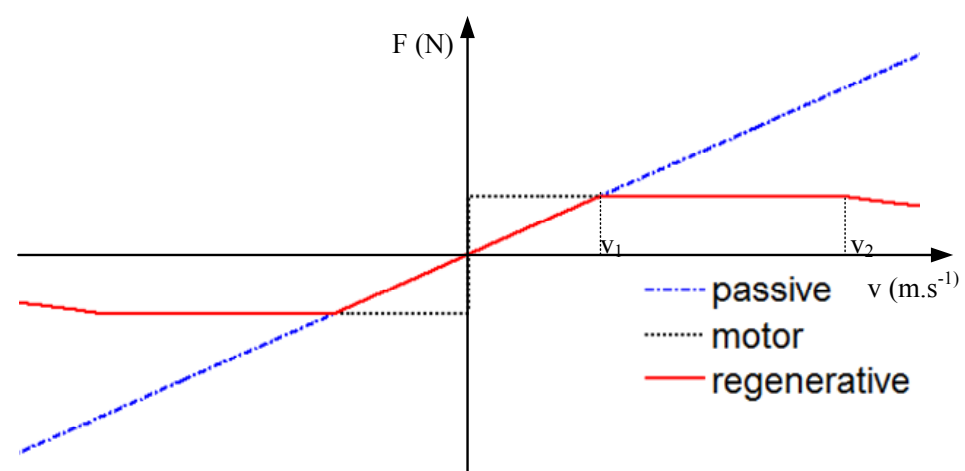

Fig. 2 Regenerative damper characteristic compared with passive damper and motor curve 
There are two objects of this damper characteristic. One is achieve high damping ratio around the resonant frequency to suppress sprung mass resonant peak response. The other is produce low damping ratio at high frequencies to isolate high frequencies vibration by utilizing the motor constant torque region. The value of velocity $v_{1}$ is the core of regenerative damper. Different set values of $v_{1}$ will lead to different transfer functions and different damping characteristics.

\section{Estimate of Initial Value of $v_{1}$}

An initial estimate of the initial value of $v_{1}$ can be obtained from the linear system's response to harmonic excitation.

Harmonic excitation is defined as: $q=A^{*} \sin (\omega t)$, where $\omega$ and $A$ are the frequency and amplitude of harmonic excitation respectively, and damping ratio $s=c /(2 \sqrt{\mathrm{km}})$, frequency ratio $\lambda=\omega / \omega_{0}$, where natural frequency $\omega_{0}=\sqrt{k / m}$.

The $H(j \omega)_{z \sim q}$ amplitude-frequency characteristic can be obtained referring to [5]:

$$
H(j \omega)_{z \sim q}=\frac{z}{q}=\frac{1+2 j \varsigma \lambda}{\left(1-\lambda^{2}\right)+2 j \varsigma \lambda}
$$

Then the response of relative velocity to excitation displacement can be obtained:

$$
\left|H(j \omega)_{(\dot{z}-\dot{q}) \sim q}\right|=\left|\frac{\dot{z}-\dot{q}}{q}\right|=\omega\left|\frac{z-q}{q}\right|=\omega\left|\frac{z}{q}-1\right|=\omega \lambda^{2}\left[\frac{1}{\left(1-\lambda^{2}\right)^{2}+(2 \varsigma \lambda)^{2}}\right]^{\frac{1}{2}}
$$

Then:

$$
|\dot{z}-\dot{q}|=A\left|H(j \omega)_{(\dot{z}-\dot{q}) \sim q}\right|
$$

When $\omega=\omega_{0}$, the relative speed of sprung mass under resonant frequency is as follows:

$|\dot{z}-\dot{q}|_{\omega=\omega_{0}}=A k / c$

In order to achieve effective vibration isolation the initial value is selected as:

$\left(v_{1}\right)_{\text {initial }}=\varepsilon(A k / c)$

Where, $\varepsilon$ is tuning factor of regenerative damper

\section{Simulation Results}

The vibration transmissibility is obtained by computing the ratio of the amplitude of steady state response of SDOF model including four different damper models mentioned above to that of harmonic base excitation. The displacement, velocity and acceleration transmissibility of sprung mass, and the relative displacement and relative velocity transmissibility of damper are investigated.

The parameters used in simulation are listed as follows:

Table 1 Simulation Parameters of SDOF Model

\begin{tabular}{|c|c|c|c|}
\hline Symbol & Value & Symbol & Value \\
\hline$m[\mathrm{~kg}]$ & 65 & $\lambda$ & 0.5 \\
\hline$k[\mathrm{~N}]$ & 4000 & $A[\mathrm{~m}]$ & 0.05 \\
\hline$c\left[\mathrm{~N} . \mathrm{s}^{-1}\right]$ & 800 & $v_{2}\left[\mathrm{~m} . \mathrm{s}^{-1}\right]$ & 1.5 \\
\hline
\end{tabular}




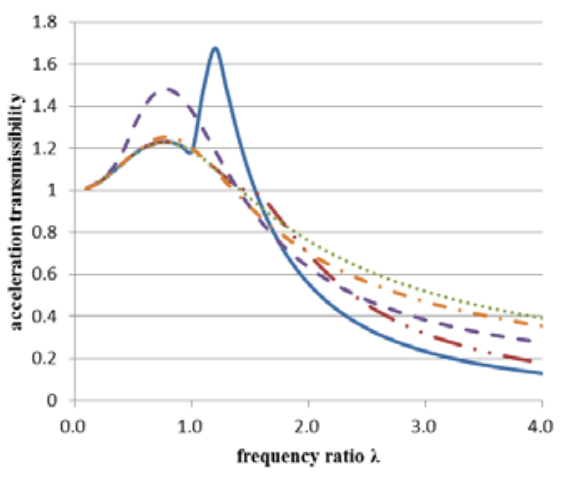

(a)

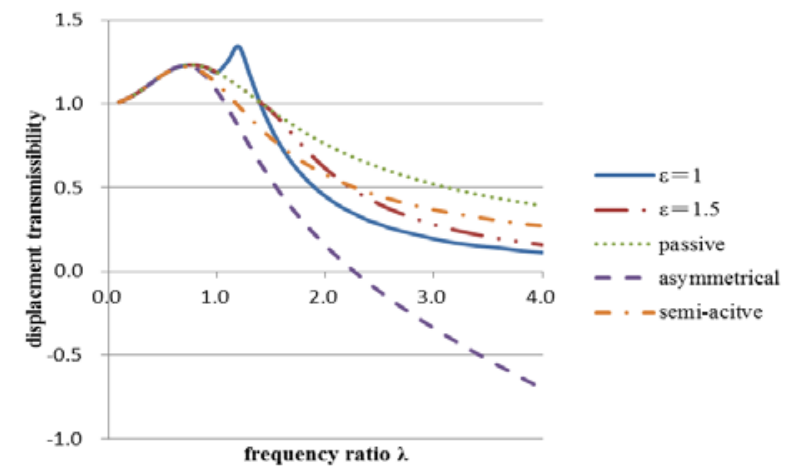

(c)

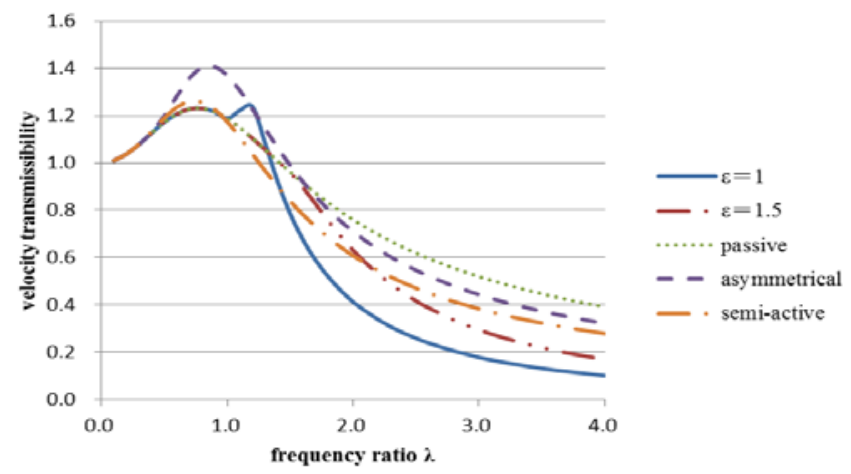

(b)

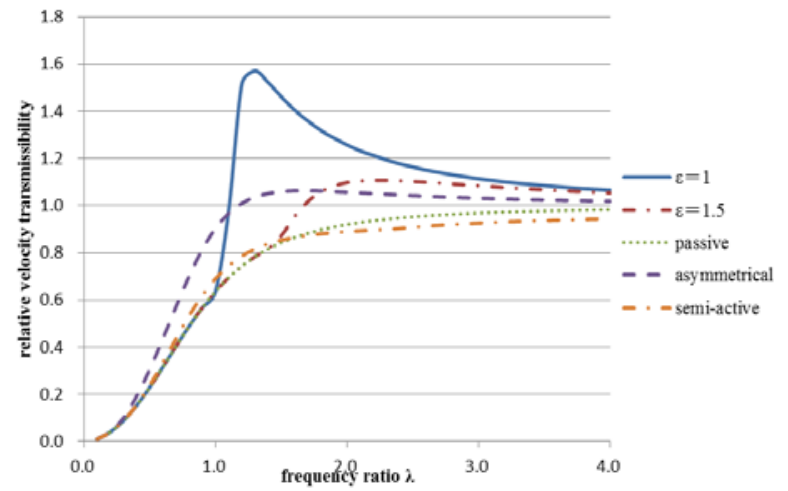

(d)

Fig. 2 SDOF model simulation results for different dampers

The acceleration and velocity transmissibility characteristics of semi-active and regenerative damper are almost the same as the passive damper for frequencies below the resonant frequency, and are superior to that of asymmetrical damper. The reason is that the equivalent damping coefficient of asymmetrical damper is smaller than that of passive damper, and smaller damping coefficient will lead to larger transmissibility near resonant frequency. The displacement transmissibility decreases even to minus values in high frequencies for the reason that the asymmetrical damper will lead to a drift to static equilibrium position as excitation frequency increases. The transmissibility characteristic of semi-active damper is superior to that of passive damper.

For $\varepsilon=1$, that means the $v_{1}$ of regenerative damper is the relative velocity response under resonant frequency calculated by Eq. 10, another peak value will appear and the transmissibility characteristic will deteriorate than other damper. When $\lambda>1$, the regenerative damper force will keep a constant value. A relatively small value of damping force near resonant frequency will lead to rapidly increase of relative velocity as illustrated in Fig. 2 (d) and thus yield large acceleration, velocity and displacement transmissibility response. For $\varepsilon=1.5$, this phenomenon disappears. The response is identical to passive damper under $\lambda<\sqrt{2}$ and in high frequencies, the acceleration, velocity and displacement transmissibility are superior to that of asymmetrical, semi-active and passive damper and litter higher than that of $\varepsilon=1$.

Therefore the value set of $v_{1}$ is very important for regenerative damper characteristic. In order to suppress the peak value near resonant frequencies, $v_{1}$ of regenerative damper should be set as the relative velocity response value near $\sqrt{2} \omega_{0}$ excitation frequencies.

\section{Summary}

As compared to passive, asymmetrical, and semi-active damper, the vibration characteristics of proposed regenerative damper has advantages for SDOF vehicle model in interesting frequency regions. 


\section{References}

[1] Songshan Liu, Hao Wei, Weihua Wang. 2011 International Conference on Electronic and Mechanical Engineering and Information Technology (Harbin, China, Aug. 1435-1439, 2011), published.

[2] Chenyuan Wang, Jiakuang Xia and Yibiao Song: Motor control technique of electric machines (China Machine Press, Beijing 2010).

[3] Hong Su, S. Rakheja and T.S. Sankar: Mechanical Systems and Signal Processing, 1989 3(1), pp.71-86.

[4] Karnopp, D., Crosby, M. J., and Harwood, R. A. ASME, 1974 vol.96, pp.619-626.

[5] Zhisheng Yu: Theory of automobile (China Machine Press, Beijing 2007). 